\title{
Mean Curvature and Asymptotic Volume of Small Balls
}

\section{Dominique Hulin and Marc Troyanov}

The aim of this note is to prove an asymptotic formula relating the mean curvature of a hypersurface at a given point and the volume of small balls centered at this point. For instance, consider a sphere $S_{R}$ of radius $R$ in Euclidean three-space $\mathbb{R}^{3}$. If $p$ lies on $S_{R}$ and $t>0$ is small enough (i.e., $0<t \leq R$ ), then

$$
\frac{\operatorname{Vol}\left(B_{p}^{+}(t)\right)}{\operatorname{Vol}\left(B_{p}(t)\right)}=\frac{1}{2}-\frac{3}{16} \frac{1}{R} t
$$

where $B_{p}(t)$ is the ball of radius $t$ centered at $p$ and $B_{p}^{+}(t)$ is the portion of the ball lying inside the sphere $S_{R}$. Our goal is to show that, up to a negligible term, a similar formula holds for any hypersurface $S$ in $\mathbb{R}^{n}$, the factor $1 / R$ being replaced with the mean curvature of the hypersurface.

We briefly recall what mean curvature is. Let $S$ be a hypersurface of class $C^{2}$ in Euclidean $n$-space $\mathbb{R}^{n}$, and assume that a unit normal vector field $N: S \rightarrow \mathbb{R}^{n}$ has been chosen (this is always possible locally). It is a basic fact that the normal acceleration $\left\langle c^{\prime \prime}(t), N(c(t))\right\rangle$ of a $C^{2}$-curve $c(t)$ on $S$ depends only on its tangent vector $V(t)=$ $c^{\prime}(t)$ : indeed,

$$
0=\frac{d}{d t}\left\langle c^{\prime}(t), N(c(t))\right\rangle=\left\langle c^{\prime \prime}(t), N(c(t))\right\rangle+\left\langle c^{\prime}(t), \frac{d}{d t} N(c(t))\right\rangle,
$$

so $\left\langle c^{\prime \prime}(t), N(c(t))\right\rangle=-\left\langle V, D_{V} N\right\rangle$ (with the notation $D_{V} N=d(N(c(t)) / d t)$.

The second fundamental form of the hypersurface $S$ at a point $p$ of $S$ is the bilinear form defined on the tangent space $T_{p} S$ by

$$
\mathrm{II}_{p}(v, w)=-\left\langle v, D_{w} N\right\rangle .
$$

The previous calculation shows that $\operatorname{II}\left(c^{\prime}(t), c^{\prime}(t)\right)$ is the normal acceleration of the curve. It is not difficult to check that $\mathrm{II}_{p}$ is a symmetric bilinear form. The mean curvature $H(p)$ of the hypersurface $S$ at the point $p$ is then defined as

$$
H(p)=\frac{1}{n-1} \operatorname{Trace}\left(\mathrm{II}_{p}\right)=\frac{1}{n-1} \sum_{i=1}^{n-1} \mathrm{II}\left(v_{i}, v_{i}\right)
$$

where $v_{1}, \ldots, v_{n-1}$ is any orthonormal basis of the tangent space $T_{p} S$. (Observe that replacing the normal field $N$ by $-N$ changes the sign of the mean curvature.) The mean curvature and the second fundamental form of a surface in $\mathbb{R}^{3}$ are classical topics in geometry (see, for instance, [1, chap. 5]).

Let us now fix a ball $B_{p}(t)$ in $\mathbb{R}^{n}$ with radius $t>0$ and center $p$ on $S$. When $t$ is small enough, the hypersurface $S$ separates $B_{p}(t)$ into two connected components $B_{p}^{+}(t)$ and $B_{p}^{-}(t)$, with the convention that $N_{p}$ points towards $B_{p}^{+}(t)$. We want to relate the mean curvature of $S$ at $p$ to the ratio of the volumes of $B_{p}^{+}(t)$ and $B_{p}(t)$. 
It is clear that for small values of $t$ the volume of $B_{p}^{+}(t)$ is roughly one-half the volume of the entire ball $B_{p}(t)$; in fact, we have

$$
\operatorname{Vol}\left(B_{p}^{+}(t)\right)=\frac{1}{2} \alpha_{n} t^{n}+O\left(t^{n+1}\right)
$$

where $\alpha_{n}$ denotes the volume of the unit ball in $\mathbb{R}^{n}$. We claim: the next term of the Taylor expansion of this volume is given by

$$
\operatorname{Vol}\left(B_{p}^{+}(t)\right)=\frac{1}{2} \alpha_{n} t^{n}-\frac{1}{2}\left(\frac{n-1}{n+1} \alpha_{n-1}\right) H(p) t^{n+1}+O\left(t^{n+2}\right) .
$$

In particular, the mean curvature satisfies

$$
H(p)=2\left(\frac{n+1}{n-1} \frac{\alpha_{n}}{\alpha_{n-1}}\right) \lim _{t \rightarrow 0}\left[\frac{1}{t}\left(\frac{1}{2}-\frac{\operatorname{Vol}\left(B_{p}^{+}(t)\right)}{\operatorname{Vol}\left(B_{p}(t)\right)}\right)\right] .
$$

To prove (1), we choose an orthonormal coordinate system centered at $p$ such that $N_{p}=(0, \ldots, 0,1)$. The hypersurface is then locally defined as a graph

$$
x_{n}=f\left(x_{1}, \ldots, x_{n-1}\right),
$$

where $f$ is a smooth function satisfying $f(0)=0$ and $\partial f / \partial x_{i}(0)=0$ for $i=$ $1, \ldots, n-1$. One then easily checks that the second fundamental form at $p=0$ coincides with the Hessian of $f$ at this point. Thus for $X=\left(x_{1}, \ldots, x_{n-1}\right)$ in $\mathbb{R}^{n-1}$, we have $f(X)=\frac{1}{2} \mathrm{II}(X, X)+o\left(\|X\|^{2}\right)$.

It is convenient to begin with an analogue of estimate (1) in which balls are replaced by cylinders. Namely, we set

$$
C(t)=\left\{x=\left(X, x_{n}\right) \in \mathbb{R}^{n-1} \times \mathbb{R}: \max \left(\|X\|,\left|x_{n}\right|\right) \leq t\right\} .
$$

If $\rho>0$ is small enough, then $f$ is well defined on $\left\{X \in \mathbb{R}^{n}:\|X\| \leq \rho\right\}$ and we consider the intersection $C^{+}(t)=\left\{x=\left(X, x_{n}\right) \in C(t): x_{n} \geq f(X)\right\}$ of the cylinder $C(t)$ with the epigraph of $f$, where $0 \leq t \leq \rho$. We then have

$$
\operatorname{Vol}\left(C^{+}(t)\right)=\frac{1}{2} \operatorname{Vol}(C(t))-\int_{B^{n-1}(t)} f(X) d X,
$$

where $B^{n-1}(t)=\left\{X \in \mathbb{R}^{n-1}:\|X\| \leq t\right\}$. Now observe that for $i, j=1, \ldots, n-1$ such that $i \neq j$ we have

$$
\int_{B^{n-1}(t)} x_{i} x_{j} d X=0
$$

while for $i=1, \ldots, n-1$

$$
\int_{B^{n-1}(t)} x_{i}^{2} d X=\frac{1}{n-1} \int_{B^{n-1}(t)}\|X\|^{2} d X=\left(\frac{1}{n+1} \alpha_{n-1}\right) t^{n+1} .
$$

Combining these three identities, we obtain 


$$
\operatorname{Vol}\left(C^{+}(t)\right)=\frac{1}{2} \operatorname{Vol}(C(t))-\frac{1}{2}\left(\frac{n-1}{n+1} \alpha_{n-1}\right) H(p) t^{n+1}+O\left(t^{n+2}\right)
$$

since $f(X)=\frac{1}{2} \mathrm{II}(X, X)+o\left(\|X\|^{2}\right)$ and $H(p)=\operatorname{Trace}\left(\mathrm{II}_{p}\right) /(n-1)$.

We still have to verify that replacing cylinders with balls does not affect the significant term in our Taylor expansion, i.e., we need to show that

$$
\left(\operatorname{Vol}\left(C^{+}(t)\right)-\frac{1}{2} \operatorname{Vol}(C(t))\right)-\left(\operatorname{Vol}\left(B^{+}(t)\right)-\frac{1}{2} \operatorname{Vol}(B(t))\right)
$$

is at most of the order $t^{n+2}$. We in fact obtain a better estimate. For any $c$ in $\mathbb{R}$ set

$$
A_{c}(t)=\left\{x=\left(X, x_{n}\right) \in C(t) \backslash B(t): x_{n} \geq c t^{2}\right\}
$$

in particular,

$$
\operatorname{Vol}\left(A_{0}(t)\right)=\frac{1}{2} \operatorname{Vol}(C(t))-\frac{1}{2} \operatorname{Vol}(B(t))
$$

The volume of $A_{-c}(t) \backslash A_{c}(t)$ is of the order $t^{n+4}$, since this set is contained in a cylinder of height $2 c t^{2}$ whose base is an annulus of outer radius $t$ and inner radius roughly $t-c^{2} t^{3} / 2$. More precisely,

$$
A_{-c}(t) \backslash A_{c}(t) \subset\left\{x=\left(X, x_{n}\right)\left|\sqrt{t^{2}-c^{2} t^{4}} \leq\|X\| \leq t,\right| x_{n} \mid \leq c t^{2}\right\} ;
$$

hence

$$
0<\operatorname{Vol} A_{-c}(t)-\operatorname{Vol} A_{c}(t) \leq 2 \alpha_{n-1} c t^{2}\left(t^{n-1}-\left(\sqrt{t^{2}-c^{2} t^{4}}\right)^{n-1}\right)=O\left(t^{n+4}\right) .
$$

Because $A_{-c}(t) \subset A_{0}(t) \subset A_{c}(t)$, we thus obtain

$$
\operatorname{Vol}\left(A_{c}(t)\right)=\operatorname{Vol}\left(A_{0}(t)\right)+O\left(t^{n+4}\right)=\frac{1}{2} \operatorname{Vol}(C(t))-\frac{1}{2} \operatorname{Vol}(B(t))+O\left(t^{n+4}\right)
$$

for all $c$. The same estimate also holds for $\operatorname{Vol} A_{-c}(t)$.

If we now choose the positive constants $c$ and $\rho>0$ so that $|f(X)| \leq c\|X\|^{2}$ whenever $\|X\|<\rho$, then we have for $t \leq \rho$ :

$$
A_{-c}(t) \subset C^{+}(t) \backslash B^{+}(t) \subset A_{c}(t) .
$$

In this way we arrive at

$$
\operatorname{Vol}\left(A_{-c}(t)\right) \leq \operatorname{Vol}\left(C^{+}(t)\right)-\operatorname{Vol}\left(B^{+}(t)\right) \leq \operatorname{Vol}\left(A_{c}(t)\right),
$$

and the previous estimates implies that

$$
\operatorname{Vol}\left(C^{+}(t)\right)-\operatorname{Vol}\left(B^{+}(t)\right)=\frac{1}{2} \operatorname{Vol}(C(t))-\frac{1}{2} \operatorname{Vol}(B(t))+O\left(t^{n+4}\right) .
$$

It is now clear that the estimate (1) follows from (3). 
The notions of the second fundamental form and the mean curvature both extend without substantial modification to the setting of hypersurfaces in Riemannian manifolds (see, for instance, [2, pp. 132-142]). We conclude this note by showing that the Taylor expansion (1) still holds in this context.

Let $S$ be a hypersurface in a Riemannian manifold $\left(M^{n}, g\right)$, and let $N$ be a (local) unit normal vector field defined in a neighbourhood of the point $p$ on $S$. The exponential map $\phi=\exp _{p}: U \subset T_{p} M \rightarrow M$ is well defined in a neighbourhood $U$ of 0 in $T_{p} M$. Moreover, when $t>0$ is small enough, $\phi$ is a diffeomorphism from the Euclidean ball $\widetilde{B}_{0}(t)$ of radius $t$ and center 0 in $T_{p} M$ to the Riemannian ball $B_{p}(t)$ in $M$. Denote by $\widetilde{S}$ the Euclidean hypersurface $\left(\left.\phi\right|_{U}\right)^{-1}(S)$ and by $\widetilde{N}$ the vector field $\left(\phi^{-1}\right)_{*} N$.

From the fact that the 1-jets of the pull-back metric $\phi^{*} g$ and of the Euclidean metric $g_{p}$ on $T_{p} M$ coincide at the origin (see [2, Proposition 5.11, p. 78]), we infer that:

(i) the mean curvature $H(p)$ of $S$ at $p$, and $\widetilde{H}(0)$ of $\widetilde{S}$ at the origin (with respect to the normal $\widetilde{N}$ ) are equal;

(ii) $\operatorname{Vol}_{\mathrm{E}}\left(\widetilde{B}_{0}(t)\right)=\operatorname{Vol}_{g}\left(B_{p}(t)\right)+O\left(t^{n+2}\right)$ (and similarly for $\widetilde{B}_{0}^{+}(t)$ and $\left.B_{p}^{+}(t)\right)$.

It follows that the formula (1) is still valid in the Riemannian setting without any correction.

\section{REFERENCES}

1. B. O'Neill, Elementary Differential Geometry, Academic Press, New York 1966.

2. J. M. Lee, Riemannian Manifolds: An Introduction to Curvature, Springer-Verlag, New York, 1997.

Département de Mathématiques, Université de Paris-Sud, 91405 Orsay Cedex - France dominique.hulin@math.u-psud.fr

Institut de Mathématiques École Polytechnique Féderale de Lausanne, 1015 Lausanne - Switzerland marc.troyanov@epfl.ch

\section{Timing Is Everything: The French Connection}

\section{W. Groetsch}

1. INTRODUCTION. Marin Mersenne (1588-1648) is remembered chiefly as a one-man scientific clearing house - the Internet of his day. During the first half of the seventeenth century, when mathematical physics was taking its first tentative steps guided by Descartes and Galileo, "Mersenne did more to propagate emerging new sciences of acoustics, pneumatics, and ballistics than anyone else of his time" [4, p. 20]. But Mersenne was more than just a go-between and publicist. He also performed "careful experiments" [5, p. 186] to test some of the new theories. Galileo's parabolic model for the ballistic trajectory of a particle in a resistanceless medium was one of the "hot" new theories of the time. This model, which is featured in nearly every elementary calculus textbook, implies that the ascent time and descent time 